\title{
A New Technique of Position-Fixing for Site Vehicles
}

\author{
Barry Gorham \\ Industrial Metrology Research Unit, University of East London, Dagenham, Essex. UK.
}

\begin{abstract}
This paper reviews the development of an automatic laser theodolite LASERFIX first designed at the University of East London in 1979 and since that time protected by a number of international patents. Applications of that theodolite to surveying, robot calibration and tunnelling machine guidance are introduced, and the specific problems of 3dimensional guidance of site vehicle are examined. Practical problems and performance capability for both off-vehicle and on-vehicle location of the laser theodolite is discussed. In both cases the design requirements for laser beam sensors or beacons is analysed and practical solutions proposed. Finally, a new receiver version of LASERFIX is introduced which does not require the use of lasers but which offers similar capabilities and performance. The paper concludes with a report of experimental tests of the new device aimed at assessing it's capability in the position-fixing of site vehicles.
\end{abstract}

\section{INTRODUCTION}

The navigational requirements of site vehicles vary according to the nature of the operations they are to perform and to the degree with which they are provided internally with local sensors and control systems. An earth-scraper, for example, could be operated more efficiently if provided with real-time spatial position co-ordinates together with three orthogonal rotation vectors. An appropriate on-board intelligent control system could then enable the production of required ground shape as an end product of an overall CAD system. Not only could the specified ground shape and geometry be produced but the process of it's production could be optimised.

A ground compactor, on the other hand, would not be able to benefit to the same extent by provision of quality information on the ' $Z$ ' parameter - local height above site datum. Here, it would be essentially 2-dimensional information which would be needed to a precision 
dictated by the operating specification, and perhaps some information on the left-right trajectory component, usually called 'yaw'.

In addition to the differing requirements of automated site vehicles for spatial information, there are also practical differences in the required rates of acquisition of that information. Thus any spatial measurement system which is used for controlling the operation of site vehicles should be capable of meeting a performance specification defined in terms of; (i) spatial parameters measured, (ii) accuracies of those parameters, (iii) measurements data acquisition rate, and (iv) system reliability.

Earlier methods of obtaining 2-dimensional position information for site vehicles relied on the use of alignment lasers for defining a linear trajectory and a dead reckoning system for determining distance along the track. This was sometimes supplemented by input from a locally established rotating laser level which provided the ' $Z$ ' component to yield 3dimensional information. A more recent 2-D system was developed by Gorham \& McCleod $^{(1)}$ which used a single vertical laser fan-beam continuously scanned about the vertical axis of the projector mounted on the vehicle in combination with an array of glass retro-reflectors situated at the edges of the site. Tilt sensors were here needed on the vehicle to compensate for it's tilt in making the plane resection calculations of position. A similar restriction applied to the CAPSY ${ }^{(2)}$ system designed essentially for 2-D position fixing.

Currently, research into the use of differential GPS for vehicle position measurement is being undertaken in many institutes around the world. Performances in all weather conditions are now being achieved which offer accuracies in measurement of ' $X$ ' and ' $Y$ ' in the centimetric range and in ' $Z$ ' in the millimetric range. Vehicle spatial attitude parameters must be derived from on-board tilt sensors and screening of the satellites from the receivers, by buildings and structures close to the vehicle, still, and predictably will continue to, limit the availability of the system.

Despite the considerable advantages of DGPS, there is still scope for development of optical systems for determining the spatial position and orientation of site vehicles in almost realtime. A comprehensive review of both two and three-dimensional optical triangulation techniques has been presented by Peyret ${ }^{(3)}$ and, in this connection, the present paper introduces two optical instruments which offer practical solutions to the problems of vehicle position and orientation measurement. They have both been developed by the Industrial Metrology Research Unit at the University of East London which has been designing instruments which exploit the geometrical properties of shaped laser beams for spatial measurements since 1977.

\section{LASERFIX}

This automatic electronic theodolite was first designed in 1979 and in respect of this device both national and international patents are currently in force from that time. 
The essential features of this instrument are that by means of a continuous scanning compound laser beam and single angle scale, the full spatial vector from the instrument to a remote point target may be determined automatically and in almost real-time.

The principle of measurement employed depends on the shaping of two laser beams which are mutually inclined to the vertical and form a fixed pair projected from a single rotary scanner. The geometry can best be described by the appearance of the pair of laser beams when intercepted by a vertical wall or screen. The beam geometry is shown below looking out from the scanner centre.

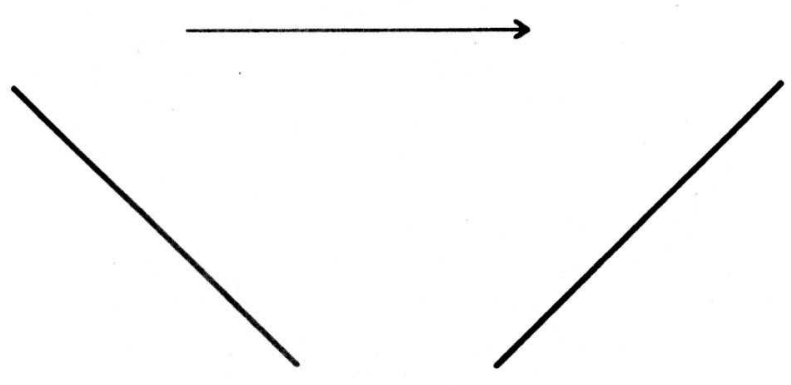

Fig. 1 The Beam Geometry of LASERFIX

The other necessary ingredient for the scanner to convert it into a theodolite is the incorporation of an electronic angle encoder into the rotor shaft. This takes the form of a glass moiré-fringe digitiser disc which generates an electrical signal for every 6 arc minutes of rotation. Sufficient inertia is built into the system to allow time interpolation between angle markers giving a precision of 1 arc second. For the vertically set scanner of this discussion, the horizontal rays in the two beams have a fixed included angle ' $\theta$ ' say, and the nominal $45^{\circ}$ tilt angle of the two beams has an actual value of ' $\gamma$ '. Both $\theta$ and $\gamma$ are determined by initial instrument calibration and remain sensibly fixed thereafter.

If an electronic photocell were to be located on the horizon through the centre of the scanner projector, say a quadrant form cell, and the angle disc in the scanner latched at the moment the sweeping first beam crossed the cell centre, a disc direction reading $\mathbf{R}_{1}$ would be generated. A corresponding reading $\mathbf{R}_{2}$ for the second beam would also be produced. Since the photocell is in the horizon plane of the scanner it is clear that $\mathbf{R}_{\mathbf{2}}-\mathbf{R}_{\mathbf{1}}=\boldsymbol{\theta}$.

Where the photocell is above the level of the local horizon, $\mathbf{R}_{\mathbf{2}}-\mathbf{R}_{\mathbf{1}}>\boldsymbol{\theta}$ and where it is below, the angular separation of those parts of the two beams which consecutively strike the photocell are closer together, and so $\mathbf{R}_{\mathbf{2}}-\mathbf{R}_{\mathbf{1}}<\boldsymbol{\theta}$. 
By knowing the value of the system constants ' $\theta$ ' and ' $\gamma$ ' and by examining the magnitude of the difference between the two latched disc readings and comparing this with ' $\theta$ ', the corresponding angle of inclination or declination of the photocell target from the laser scanner can be determined. The mean of the two beam readings $\mathbf{R}_{\mathbf{1}}$ and $\mathbf{R}_{\mathbf{2}}$ is used to determine the azimuth of the target. Full spatial direction measures to all suitable point targets within the field of illumination of the scanner can thus be obtained for every full circle rotation of the scanner. The rate of direction data update is limited largely by the speed of the electronic counters used in the interpolation of the moiré disc pulses. A reasonable practical limit is close to $50 \mathrm{~Hz}$ for the scanner, although this may have to be reduced due to limitations of photocell detection of scanning beams at long ranges.

There are three kinds of targets which can be used with this instrument; photocells which enable direct identification of the target for the purposes of correlating with the associated disc readings; passive retro-reflectors which do not lend themselves to ready identification, and active reflectors or transponders which may be coded by a number of techniques to identify themselves to the system computer at the location of the scanner. In the cases where reflective types are used, it is necessary to mount on the scanner, photocell receivers, which are appropriately located with respect to the beam projection points so as to register the point of incidence of the centre of the beam on the centre of the reflector.

In order to use this kind of instrument, which is essentially a theodolite, for the measurement of spatial position, it is necessary to introduce some form of length scale. One way of doing this is to arrange that the targets to be used for the measurements are set into a fixed and known spatial array. The positioning process then comprises a measurement of the direction from the scanner to each individual target of the array followed by a spatial resection analysis which generates the individual 3-D co-ordinates for each target in the co-ordinate reference frame of the scanner. It was this technique which was used in the first application of LASERFIX as a robotic 'Total Station' surveying instrument, first produced in 1985 ( Gorham ${ }^{(4)}$ ); also in the automatic tunnel guidance system LASERGUIDE, first produced in 1987 ( Gorham $\left.{ }^{(5)}{ }^{(6)}\right)$, and the system for automatic in-situ spatial calibration of production robots LASERCAL, first produced in 1988 ( Gorham $^{(7)}$ ).

Another method is to simulate the well known two-theodolite intersection technique which uses two separated instruments at the ends of a baseline. The scale here is introduced by the known length of the baseline and often leads to much improved geometry for the measurements and consequently greater accuracy. This method will also work with only a single target; an advantage where attitude information for the vehicle is not required

\section{LASERGUIDE}

The application of the theodolite for the guidance of tunnelling machines (LASERGUIDE) had it's first industrial trials in $1987-8$ by British Coal. The scanner unit was mounted in the roof of the tunnel such that the pair of laser beams projected the following shape ' $>$ ' which

13th ISARC $-720-$ 
was rotated in a vertical plane. The rear of the tunnelling mole was fitted with an array of fixed glass retro-reflectors and for every sweep of the compound laser beam the co-ordinate position of the centroid of the machine together with it's three rotation parameters was computed. The performance parameters achieved during the trials over a range of 120 metres were $+/-5 \mathrm{~mm}$ in the transverse axes measurements in the tunnel; $+/-5 \mathrm{~cm}$ in measurements along the longitudinal axis, and $+/-0.05^{\circ}$ accuracy in roll, pitch and yaw.

\section{LASERTRACK}

Although LASERGUIDE was designed with a He-Ne laser, more recent versions of the theodolite instrument have been designed using an infra-red semiconductor laser source. One such instrument, which we have called LASERTRACK, has been specifically designed for site vehicle position-fixing and orientation measurements. The instrument is mounted on the vehicle and with static glass-retroprism targets the theodolite can achieve a precision of $+/-3$ arc seconds in both horizontal and vertical direction measures at a range of 100 metres and at a repetition rate of $10 \mathrm{~Hz}$. This translates into a static accuracy of $+/-1.5 \mathrm{~mm}$ in all three position co-ordinates. Research is continuing to translate these static measurements into dynamic measurements so that for a moving vehicle a plan position accuracy of better than $10 \mathrm{~cm}$ is reliably achieved, and for the ' $Z$ ' parameter, a figure of $+/-5 \mathrm{~mm}$.

Details of a suitable 3-D spatial resection technique for this instrument have already been published by Malinovsky et al. ${ }^{(8)}$ and will not be repeated here. Although Laserfix was not mentioned by name in that paper, all of the significant details of the system design were described there and so this paper will continue with some discussion on the design of suitable targets.

\section{FIELD TARGETS}

Passive retro-reflective targets are not particularly suitable for a 3-dimensional resection system since it is virtually impossible to identify a particular target without ambiguity. Their other main drawback is the need to transmit the light signal over twice the useful path. A lesser disadvantage is that the retro's field of view is typically only $+/-25^{\circ}$ and provision of a necklace of such retro's at each field target station can be prohibitively expensive. The problem can be alleviated to some extent by designing the field target with a single retro which is rotatable in azimuth by a motor driven by a simple potentiometer encoder control system. The target receives a radio signal from the vehicle containing it's local X-Y position and an integral processor moves the potentiometer and hence axis of the retro to maintain it's pointing towards the vehicle. The pointing accuracy required is only about $+/-5^{\circ}$.

Photocell detectors situated at the edges of the site usefully solve the double-path problem, but in order to produce the position information at the vehicle, it is necessary to immediately transmit the target signal back to the scanner encoder. The resulting transponders have some problems of their own. The simplest and probably least expensive method is to transmit a 
UHF radio pulse back to the vehicle scanner immediately that a field target is struck by a laser beam. However, the bandwidth requirements for such a transmission, of the order of $1 \mathrm{MHz}$, are not licensable at present anywhere in the world. Consequently, this is not a practicable solution.

At UEL we have designed a number of optical transponders, some self-pointing in the manner described above for retro-prisms, and others with a wide angular projection cone angle for smaller sites. These may be provided with a LED or semiconductor laser source. The former are much less expensive than the latter but their minimum pulse width for radiation transmission is not less than $1 \mu \mathrm{s}$, whereas the lasers can produce pulses of only $50 \mathrm{~ns}$ width if required, and at much increased radiant power. The significance of these parameters is that in practice the field target transponders are not only required to transmit their signals so that they may reliably be received by the scanner photocell, but also that the radiation which they produce does not present a hazard to the human eye.

In this connection, we have achieved a compromise between high level radiation transmission and eye safety by the use of holographic lenses. These have an optical behaviour which is similar to a high density array of adjacent individual micro-lenses and produce an output beam which is of highly uniform intensity and of closely controlled radiation pattern. The radiation output of our optical transponder is a uniform cone of fixed internal angle, typically $60^{\circ}$.

The uniformly radiating face of the $50 \mathrm{~mm}$ diameter circular disc holographic lens constitutes an extended object to the eye and thus is not capable of producing a significantly small image on the retina. It is considerably less hazardous to the eye than even a large area beam derived from a LED or laser source using conventional lenses. Accordingly, considerably greater radiant power can be transmitted from such a device without risk of causing eye injury than is possible by any other traditional form of laser projector.

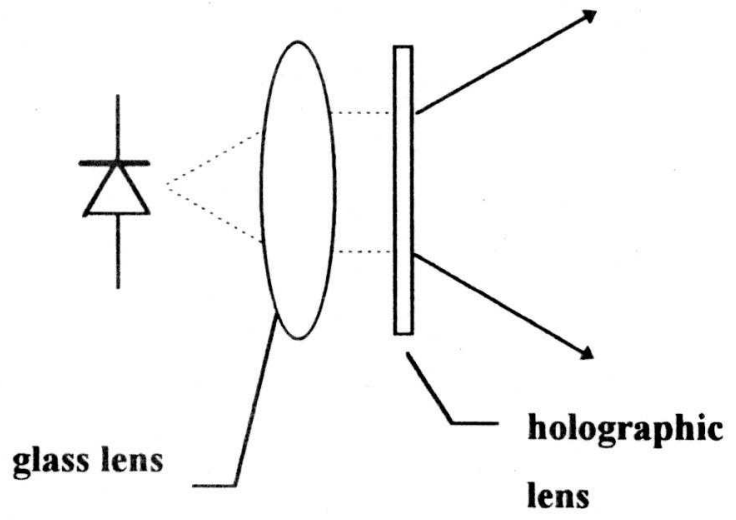

Fig. 2 Optical Transponder with a Holographic Lens 
Research into the design of eye-safe optical transponders has led to a reappraisal of the basic LASERFIX design. This is a theodolite which transmits a radiation pattern which is received at a remote point where the particular part of the pattern received is identified, and hence the direction of the line joining the instrument to the target is determined. This has led to the design of a new kind of automatic theodolite which is like LASERFIX in reverse.

\section{A NEW DESIGN OF THEODOLITE}

Like a conventional theodolite, the new instrument measures the direction of radiation it receives from a distant luminous source or target; unlike a conventional theodolite, it does not need a visible source and it measures the full direction vector to that target using only a single circular scale. The essential features of the new design are shown below in Fig. 3 .

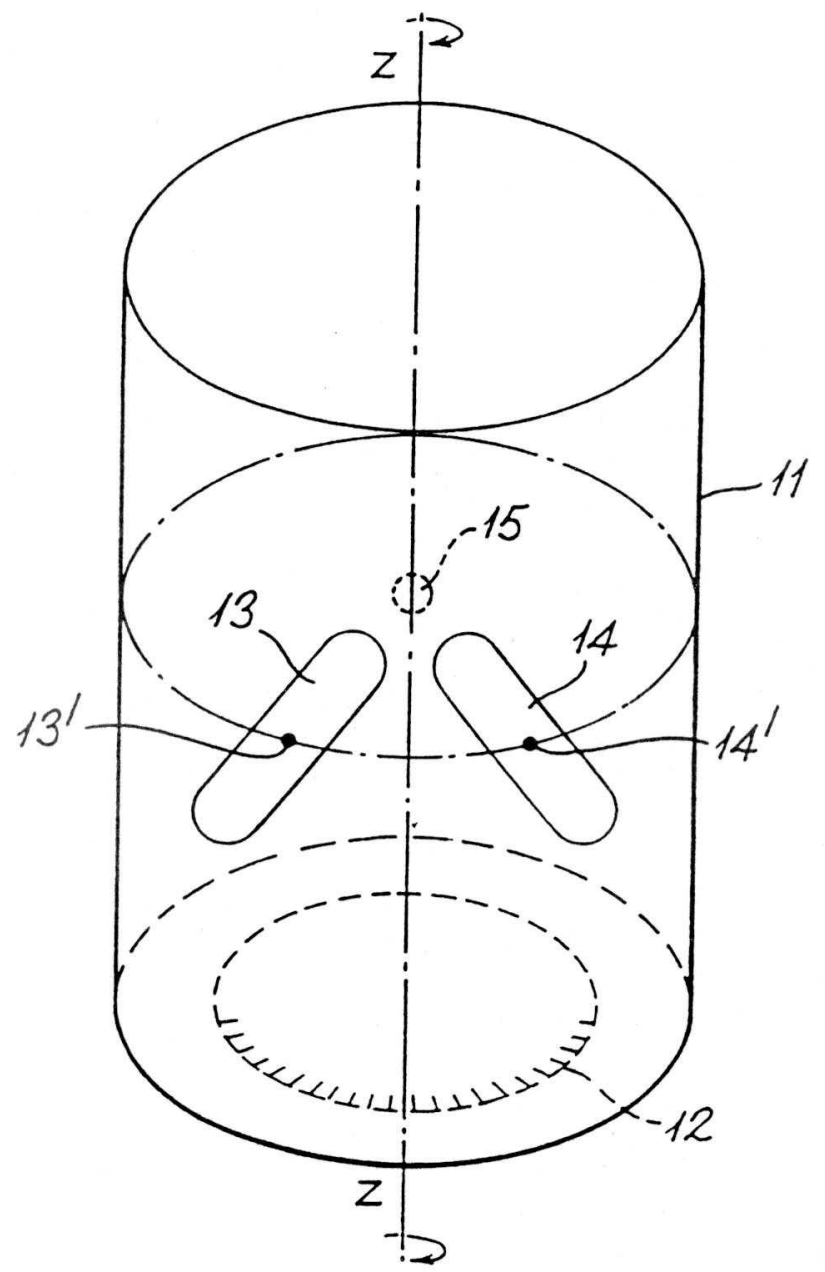

Fig. 3 Schematic of a New Automatic Theodolite 
The body of the new theodolite is shown as a cylindrical housing 11 which is mechanically rotatable about it's primary axis $\mathbf{Z}-\mathbf{Z}$, which would normally be vertical. The rotations of the housing 11 are continuously monitored by a glass disc moire fringe angle encoder 12 . In the side wall of the housing two cylindrical lenses 13 and 14 are located; these have their optical expansion axes oppositely skewed relative to the rotation axis $\mathbf{Z}-\mathbf{Z}$ so as to form a symmetrical inverted ' $V$ ' configuration.

Lines drawn through the centres, 13' and 14', of the two cylindrical lenses, and which are perpendicular to the rotation axis $\mathbf{Z - Z}$ will intersect on that axis at the point $\mathbf{1 5}$. It is here that a photocell is located with it's sensitive surface facing a point mid-way between the centres of the lenses.

Below, in Fig. 4, can be seen the relative position of a distant luminous source ' $\mathbf{S}$ ' ( assumed here to lie in a horizontal plane through the points 13' and 14'), the centres of the lenses and the photocell 15. The angular separation of the centres of the two cylindrical lenses is fixed and denoted here by ' $\theta$ '.

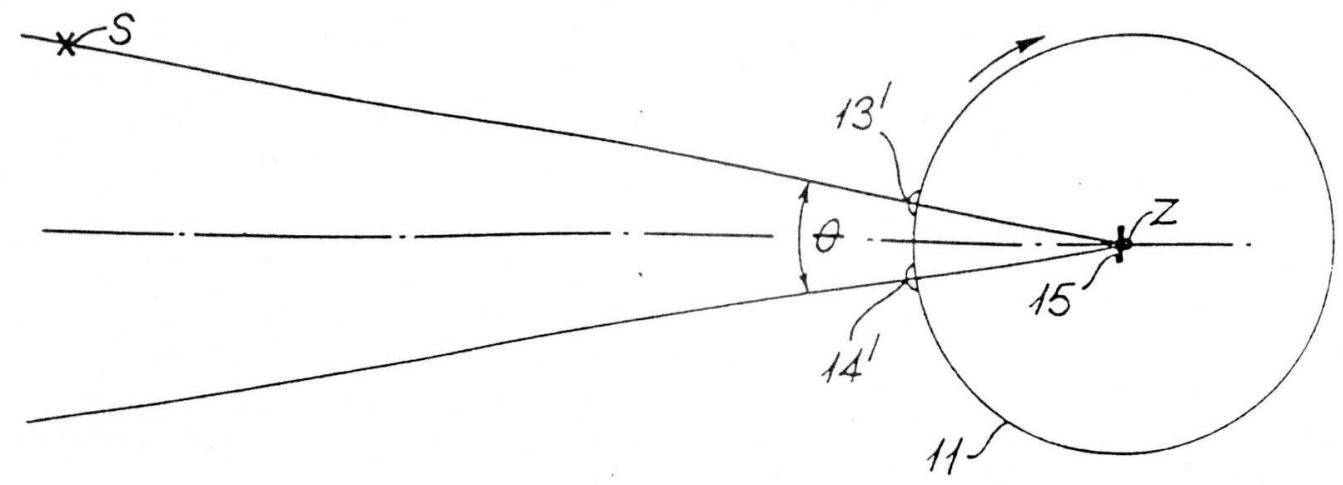

Fig. 4 Plan View of the Light Paths from Target to Theodolite

As the cylindrical housing rotates the two cylindrical lenses successively face the luminous source ' $\mathbf{S}$ ', and once the light from ' $\mathbf{S}$ ' enters a lens a line image is produced which is focused in the plane of the photocell $\mathbf{1 5}$. The two line images will lean in opposite senses with respect to the rotation axis $\mathbf{Z - Z}$, and as each one sweeps across the photocell an electrical signal is generated which latches the instantaneous reading of the angle encoder.

As before for LASERFIX, the angular separation of those parts of the line images which intercept the photocell as the instrument is rotated is determined by the inclination of the luminous source (target) to the plane (usually horizontal ) which contains the two lens centres 13' and 14'. The three cases for the line image geometry is illustrated in Fig. 5 below where it is assumed that the instrument is rotating in an anti-clockwise direction and the leading $(\mathbf{L})$ and trailing $(\mathbf{T})$ beams are successively crossing the photocell located on the instrument rotation axis. 
In the figure, diagram (i) shows the luminous source to be on the instrument horizon; (ii) shows it to be above that horizon, and (iii) shows it to be below.

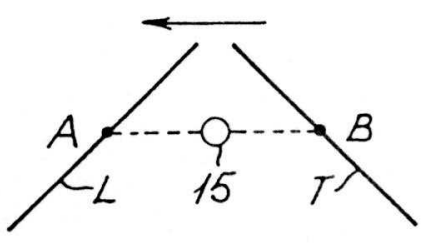

(i)

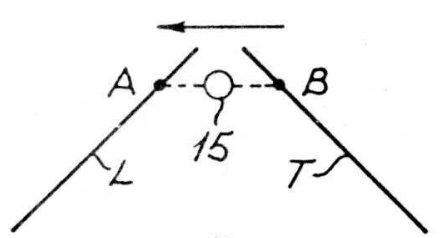

(ii)

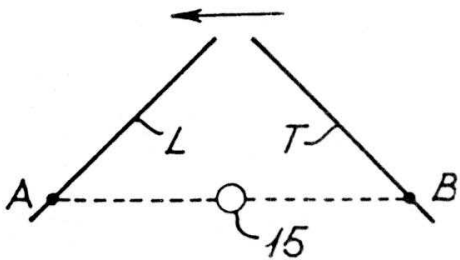

(iii)

Fig. 5 The Three Geometries for the Line Images

The latched angle values from the encoder are used as before to compute the full direction vector of each luminous source target within the field of view of the instrument. However, unlike the LASERFIX optical transponders, the radiation source here must be continuous. Consequently, and mainly for eye safety reasons, the available radiant powers which can be used are more limited.

Experiments using high power $(60 \mathrm{~mW})$ invisible LED sources in cw mode for the luminous source, and within the published ${ }^{(9)}$ eye safety limits, produced acceptable signal levels at the theodolite receiver photocell over ranges of up to 100 metres. The standard error of pointing of the instrument over a set of twenty consecutive observations is close to 6 arc seconds and this is largely a function of the quality of the quadrant photocell used. Detection of the radiation from the source can be improved by imposing high frequency modulation, preferably not less than $1 \mathrm{MHz}$ although this is possible only when semiconductor lasers or low power LEDs are used.

However, unlike LASERFIX, this instrument is capable of operation at high rotation speeds irrespective of range. Even at high rotation speeds, the rate at which the luminous line images sweep across the photocell remains modest. This is due to the small radius of the main cylindrical housing of the instrument which itself is not affected by the range of the distant luminous target.

Simplicity and low cost of this new instrument make it an attractive alternative to LASERFIX for performing the spatial measurements required for 3-D position-fixing of site vehicles. It's performance is inferior in terms of accuracy and range of operation at present, but research is currently underway to improve these features whilst exploiting it's many other practical advantages. 


\section{BIBLIOGRAPHY}

[1] Gorham B. J. and McCleod F.

Lasers in Farm Management

Land \& Minerals Surveying Journal:Research, Vol. 9 No.1 January 1991

[2] De Vos L. and Hasara B.

Field Applications with CAPSY

10th ISARC, Houston, May 1993

[3] Peyret F.

Towards the Automation of Road Work Sites - The Contribution of New Positioning

Techniques.

Internal paper. LCPC, Centre de Nantes, BP 19, 44340 Bouguenais, France. Nov.1995.

[4] Gorham B. J.

Measurement of Spatial Position using Shaped Laser Beams.

Land \& Minerals Surveying Journal:Research, Vol. 6 March 1988

[5] Gorham B. J.

Laser Guidance System for Tunnelling Machines

Land \& Minerals Surveying Journal:Research, Vol. 7 September 1989

[6] Gorham B. J.

The LASERGUIDE System of Automatic Machine Guidance.

11th ISARC, Brighton, May 1994

[7] Gorham B. J.

Automatic Calibration of Production Robots from a single Laser source.

Land \& Minerals Surveying Journal:Research, Vol. 7 January 1989

[8] Malinovsky Y. et al.

A System for determining the Localisation of Construction Equipment on the Working Site.

8th ISARC, Stuttgart, June 1991

[9] International Standard of Radiation Safety of Laser Products. IEC 825-1-2:1995. 